\title{
Somatic Mosaicism as Modulator of the Global and Intellectual Phenotype in Epimutated Angelman Syndrome Patients
}

\author{
Silvia Russo ${ }^{1, *}$, Ester Mainini ${ }^{1}$, Chiara Luoni ${ }^{2}$, Francesca Cogliati ${ }^{1}$, Valentina Giorgini ${ }^{1}$, \\ Maria Teresa Bonati ${ }^{3}$, Francesca Forzano ${ }^{4}$, Cristiano Termine ${ }^{2}$, Alessandra Murgia ${ }^{5}$, \\ Mara Patrini ${ }^{6}$, Antonella Fabretto ${ }^{7}$, Skabar Aldo $^{7}$, Elena Freri ${ }^{6}$, Vanna Pecile ${ }^{7}$ and \\ Lidia Larizza ${ }^{1,8}$
}

\author{
${ }^{1}$ Molecular Genetics Laboratory, IRCCS Istituto Auxologico Italiano, Via Zucchi, 18, 20095 Cusano Milanino, \\ Milan, Italy \\ ${ }^{2}$ Child Neuropsychiatry Unit, Department of Clinical and Experimental Medicine, University of Insubria, \\ Varese, Italy \\ ${ }^{3}$ Clinical Genetics Laboratory, IRCCS Istituto Auxologico Italiano, Piazzale Brescia 1, Milan, Italy \\ ${ }^{4}$ Medical Genetics Unit, Galliera Hospital, Genova, Italy \\ ${ }^{5}$ Laboratory of Molecular Genetics of Neurodevelopment, Department of Women's and Children's Health, \\ University of Padova, Italy \\ ${ }^{6}$ Department of Pediatric Neuroscience, Foundation I.R.C.C.S. Neurological Institute C. Besta, Via Celoria,11, \\ Milan, Italy \\ ${ }^{7}$ Institute for Maternal and Child Health, Foundation I.R.C.C.S. Burlo Garofolo Institute, Via dell'Istria 65/1, \\ 34137 Trieste, Italy \\ ${ }^{8}$ Department of Health Sciences, University of Milan, Italy
}

\begin{abstract}
Angelman Syndrome (AS) is due to the loss of function of the single UBE3A gene, mapping to chromosome $15 q 11-q 13$ and encoding the E6AP ubiquitin ligase. Expression of UBE3A is subject to genomic imprinting which is restricted to the brain, where only the maternal allele is transcribed. AS pathogenetic mechanisms include deletion of the maternal 15q11-13 chromosomal region, chromosome 15 paternal uniparental disomy (UPD), Imprinting Defects (ImpD) leading to silencing of the maternal allele and intragenic mutations of the maternal UBE3A allele. From our AS cohort we sorted out for detailed clinical-molecular characterization six mosaic cases, five with ImpD epimutations and one with patUPD15. This latter case referred for intellectual disability and fortuitously solved by SNP array, is, to our knowledge, the unique patient reported with mosaic patUPD of this imprinted region. Somatic epimutation mosaicism represents a challenge for both clinical and molecular diagnostics. The described patients, referred to our center either for uncertain AS or simply for intellectual disability, could be molecularly characterized by applying a multi-method approach including Methylation-Sensitive PCR and MS-MLPA without a strict cut off. The percentage of normal cells detected ranged up to $40 \%$. We confirm the mild phenotype reported in mosaic AS ImpD and provide a detailed analysis of IQ. Mild mental retardation, with significant difficulties in language expression, but only mildly impaired performance skills, together with pathognomonic EEG, is a cue not to overlook in mosaic AS patients. Mosaic epimutations should be searched also in patients with minor AS features and presenting only with intellectual disability.
\end{abstract}

Keywords: Mosaicism, intellectual disability, Angelman syndrome, epimutation, uniparental disomy.

\section{INTRODUCTION}

Intellectual disability (ID), intended as neurodevelopmental disorder and defined by an intelligence quotient (IQ) below 70 associated to a deficit in behavior attitude, has a reported prevalence of $1-3 \%$ in the world population [1,2]. Considering this relevant proportion of patients, syndromic and non syndromic ID forms are distinguished, according to the presence of one or more other clinical and often

*Address correspondence to this author at the Molecular Genetics Laboratory, IRCCS Istituto Auxologico Italiano, Via Zucchi, 18, 20095 Cusano Milanino, Milan, Italy; Tel: +3902619113036; Fax: +3902619113033;

E-mail: s.russo@auxologico.it specific features [3]. The expression of the phenotype of syndromic ID may be highly variable and often far from the common features of the reference syndrome. Genetic and environmental factors may concur to the heterogeneous clinical presentation. Among genetic factors, mosaicism, defined by the presence of two or more genetically different cell populations within the same individual, can account for a fraction of the commonly observed clinical variability.

Angelman syndrome (AS; \#105830), is a neurodevelopmental disorder characterized by severe to moderate intellectual disability, hyperactivity, absence of speech, ataxia, a peculiar abnormal EEG 
and a typical happy disposition. A wide number of additional features have been reported in subsets of patients. Consensus for diagnostic criteria was established in 1995 [4] and revised in 2006 [5] to detail the AS syndromic phenotype.

Abnormalities affecting the UBE3A gene, mapping within the 15q11-13 chromosomal region, are involved in the etiology of the syndrome [6-8]. The disease gene codes for the ubiquitin protein ligase E3A, implicated in protein degradation through the ubiquitin proteasome pathway, essential for cellular functioning and crucial to normal synaptic development and neural plasticity $[9,10]$. The $15 q 11-13$ region is subject to imprinting regulation and the paternal allele is silenced in neurons, while the maternal allele is expressed $[7,11]$. Four different mechanisms may impair the expression of the maternally derived gene which in order of prevalence are deletion of 5-7 $\mathrm{Mb}$ including the imprinted region on the maternal chromosome 15 $(75 \%)$, intragenic mutation of UBE3A gene $(5-10 \%)$, epigenetic alteration of the imprinting centre, ImpD (imprinting defects) (2-4\%) and paternal uniparental disomy (patUPD15) (3-7\%) [7,8]. A large body of evidence on genotype-phenotype correlation highlighted that a full phenotype is associated with deletions or UBE3A point mutations, while patUPD15 and $I \mathrm{mpD}$ are usually leading to a less impaired clinical phenotype [12-14]. The molecular diagnosis carried on by MS-MLPA detects in a semiquantitative way changes in DNA methylation and copy number, while microsatellite analysis or SNP array are needed to discriminate between Imprinting Defects and paternal uniparental disomy. Several AS mosaic are reported [15-19] and currently $10 \%$ of SNRPN methylation is considered the cut off for diagnosis, but patients with percentages up to $20 \%$ have been reported [16, 19], corresponding to a cellular mosaicism with $40 \%$ of normal cells. All the mosaic cases were caused by ImpD and accordingly showed an altered methylation pattern, but no patUPD15 has yet been reported. The phenotype of mosaic cases appeared quite atypical, with a few of them resembling Prader Willi (PWS) syndrome [15,17-19]. Only in one case ID was measured by intellective scales [19].

We herein describe six peculiar cases clinically evaluated as uncertain AS due to mild to atypical phenotype, who turned out to be low level mosaic for ImpD epimutations (five cases) and patUPD15 (one case). All were referred by neuropsychiatry clinicians for mild ID and speech delay. The last case is quite unique, because up to date only few atypical PWS patients mosaic for matUPD15 has been described [20]. The atypical patients represent a challenge for both the clinical and the molecular diagnosis and their characterization may enhance the comprehension of the wide clinical and genetic spectrum underlying Angelman syndrome.

\section{METHODS}

\section{Patients}

Cases 1, 3, 4, 5 and 6 were referred to our laboratory for the SNRPN methylation test in order to confirm/exclude the clinical suspicion of Angelman syndrome. Clinical data sheets reported mild psychomotor delay and/or learning disability, absence or delay of speech, abnormal EGG and/or seizures in all cases, although at variable degree. Conversely patient 2 was sent to our lab to confirm mosaicism for patUPD15 which has been detected by SNP array. Karyotype abnormalities and Fragile $X$ syndrome had been previously excluded in all the patients. Following the molecular diagnosis all patients were clinically reevaluated and carefully followed up. EEG and cognitive scales were subsequently requested, if available.

Evaluation of patients' intellectual abilities relied on comprehensive standardized neuropsychological assessment.

The following tests, administered according to standard procedures, were used:

\section{Wechsler Preschool and Primary Scale of Intelligence}

WPPSI-III, Wechsler Intelligence Scale for children third edition - WISC-III, Wechsler Intelligence Scale for children - fourth edition - WISC-IV, Wechsler; IQ<70 was considered the cut-off point [21].

\section{Visual-Motor Integration (Copying form Test of Developmental Test of Visual-Motor Integration - VMI)}

It measures visual-motor integration or the degree to which visual perception and finger-hand movements are coordinated. The task consists of 27 geometric forms of increasing complexity presented on paper. The child is required to copy these forms.

\section{Raven's Progressive Matrices}

The test assesses non verbal intelligence. Each of the 36 test items consists of an incomplete abstract 
pattern. Participants are required to select, from a set of six, the figure needed to complete the pattern correctly.

\section{Leiter International Performance Scales-Revised}

The Leiter-R test system consists of 20 subtests included in two different batteries: a) the visual function and reasoning battery, measuring non-verbal intelligence in the form of visual functioning, reasoning and visuo-spatial skills, and b) the memory and attention battery, which are used to evaluate nonverbal performance in memory and attention domains [22].

\section{Griffiths Mental Development Scales 2-8 (GMDS 2-8)}

The test measures six developmental domains: locomotor (gross motor), personal social (self-care and social interaction), hearing and language, eye-hand co-ordination performance (form perception, modelmaking, visual-spatial reasoning) and practical reasoning (dimensions, digit span, sequential reasoning) and an overall developmental quotient. Standardized developmental subquotients for developmental domains were created and an overall development calculated by averaging the developmental subquotients.

A classification of delay was given at each assessment basing on DSM-5 criteria (average 86115 , low average $71-85$, mild delay $56-70$, moderate delay $41-55$, severe delay $26-40$, profound delay $\leq 26$ ) [23].

\section{Vineland Adaptive Behavior Scale (VABS), Interview Edition}

It is a semi-structured interview conducted with the child's primary caregiver. It assesses a child's ability to perform activities of daily living required for personal and social competence. It yields standard scores and equivalent age in four domains including communication, daily living skills, socialization, and motor skills (the last only for children under 6 years of age) [24].

\section{Test for Reception of Grammar (TROG-2)}

This test assesses the receptive language, particularly the understanding of grammatical contrasts. Each test stimulus is presented in a four picture multiple-choice format, one of which corresponds to the short sentence spoken by the examiner, whereas the rest are lexical and/or grammatical foils.
EEG

The EEG was recorded using $\mathrm{Ag} / \mathrm{AgCl}$ electrodes placed according to the International 10-20 system; polygraphic signals included electro-oculogram (EOG), pneumogram, and additional surface electro-myogram (EMG) including at least the deltoid muscles, if indicated. Moreover, time-locked video monitoring was associated when appropriate. Signals were acquired by a computerized Micromed system. We evaluated the EEG signal's organization while awake and asleep and the morphology, localization, occurrence and incidence of any paroxysmal and/or epileptiform anomalies, according to the International Guidelines of Neurophysiology.

\section{Chromosome Analysis}

Cytogenetic and molecular cytogenetic studies were performed on chromosomes derived from peripheral blood according to standard procedures. QFQ-banded slides with a resolution of 400-500 bands were employed for chromosome spreads analysis. SNP array analysis, from DNA isolated from peripheral blood lymphocytes, was carried out using the Illumina HumanOmniExpress genotyping microarray according to the protocol. Data analysis was performed using the Illumina GenomeStudio v.2010 software.

\section{MS-PCR SNRPN}

Genomic DNA was treated with sodium bisulfite using the EZ DNA MethylationTM Kit (Zymo Research). Methylation-specific PCR analysis of the SNURFSNRPN exon 1/promoter was performed using two specific primer pairs, one specific for the methylated maternal allele and the other for the unmethylated paternal allele [25]. The SNRPN-M forward (taaataagtacgtttgcgcggtc) and SNRPN-M reverse (aaccttacccgctccatcgcg) primers amplify a region of $174 \mathrm{bp}$ on the methylated maternal chromosome 15 , while the SNRPN-P forward (gtaggttggtgtgtatgtttaggt) and SNRPN-P reverse (acatcaaacatctccaacaacca) primers were used for the amplification of a $100 \mathrm{bp}$ region within the unmethylated $\mathrm{CpG}$ island of the SNRPN gene promoter. In both PCRs, the number of PCR cycles was adjusted to stop the reaction before the plateau phase to allow even low grade mosaicism to be detected.

\section{MS-MLPA at SNRPN locus}

MS-MLPA was performed using Salsa MS-MLPA Kit ME028 PWS/AS (MRC-Holland-Amsterdam, The 
Netherlands) according to the kit instructions. DNA was processed in parallel with and without digestion with methylation sensitive Hhal enzyme to detect both methylation pattern and copy number variation (CNVs) in the Prader-Willi/Angelman critical region on chromosome 15q11, in a semiquantitative manner. Data analysis was carried out by the Coffalyser.net software (Software version: v131211) which provides two outputs: one related to CNVs and the other to the methylation status. The methylation status of SNRPN locus is the average of methylation ratio of single SNRPN MLPA probes. The methylation ratio of each MLPA probe is the ratio of the output from methylation and CNVs analysis. Each test sample is referred to positive and negative references (in the ratio of one negative control every seven cases).

\section{Microsatellite Analysis}

The following 16 polymorphic loci spanning the whole chromosome 15 were selected from the $A B I$ Prism Linkage Mapping Set: D15S541, D15S18, D15S912, D15S11, D15S128, D15S122, D15S10, D15S986, D15S97, GABRB3, D15S511, D15S1002, D15S165, D15S1012, D15S153 and D15S205. Markers from D15S541 to D15S165 map within 15q1113, while markers D15S1012, D15S153, D15S205 are located outside the critical region and used as controls. PCR cycles number were adjusted to disclose even low grade pat15UPD mosaicism. PCR fragments were separated by capillary electrophoresis on the automated $\mathrm{ABI} 310$ sequencer and data analysed matching parental to proband transmission. Mosaicism occurrence and level were assessed by the ratio between maternal and paternal peak areas.

\section{RESULTS}

Since 1995 a total of 1020 patients with a clinical presentation raising the suspicion of Angelman syndrome were referred to our laboratory by neuropediatricians. A set of 158 out of 1020 patients $(15,5 \%)$ aged from 5 months to 60 years, achieved positive molecular diagnosis. Genetic testing by a flow chart including 15q11-13 region methylation test and UBE3A sequencing identified 84 deletion carriers, 9 patUPD15, $18 \mathrm{ImpD}$, and 47 UBE3A mutated cases. According to the literature $[7,13]$ our deletion patients showed a very representative AS phenotype, those carrying UBE3A mutations were clinically heterogenous depending on the type of mutation, while those with patUPD15 and ImpD displayed a comparatively milder expression of the disease. The six AS patients herein described fall within the two latter molecular groups, although presenting with molecular features accounting for their subtle and atypical clinical phenotype.

\section{Molecular Characterization of Mosaic ImpD and patUPD15 AS Cases}

Methylation test, carried on by MS-PCR and MSMLPA on cases 1, 3, 4 and 5 (Figure 1A) and only by MS-PCR on case 6 (Figure 1B) highlighted the occurrence of mosaic ImpD epimutations in five cases out of the overall $18(28 \%)$ AS patients with ImpD. The ratio between methylated $\mathrm{CpG} /$ methylated plus unmethylated $\mathrm{CpG}$ at the SNRPN locus obtained by Coffalyser analysis showed a variable degree of SNRPN methylation status ranging from 0,2 for the patient with the highest level of mosaic epimutation to 0,035 for the patient with only a low number of correctly methylated paternal $\mathrm{CpGs}$ (see the values in Figure 1A). As reported at the bottom of Figure 1A, the corresponding percentage of normal cells in blood varies from 41 to $7 \%$, in agreement with data observed by Nazlican et al. (2004) [16]. Segregation analysis of polymorphic markers on chromosome 15 excluded the occurrence of paternal uniparental disomy, in all cases except case 2 .

In patient 3 we also evaluated the SNRPN methylation status in the DNA from buccal swab. Methylation values in this tissue perfectly overlapped those obtained on blood (data not shown).

Patient 2 underwent molecular karyotyping by highresolution SNP arrays, which revealed the presence of complete isodisomy of chromosome 15 (Figure 2A). The percentage of isodisomy calculated from $B$ allele frequencies ranged from 40 to 50 . MS-MLPA (Figure 1A) and microsatellite analyses (Figure 2B) confirmed the occurrence of paternal isodisomy with a percentage of methylation of $19,5 \%$, accounting for a percentage of normal cells of $39 \%$.

\section{Clinical Features of Mosaic ImpD and patUPD15 Cases}

Table 1 shows the clinical features of the mosaic patients. Traits which did not support the suspicion of Angelman syndrome are the late age of definite diagnosis, which was 6 years in 4 out of 6 children, the early age of walking, from 10 to 18 months, the very mild ataxia only observed in 2 out of 6 patients and a good level of general comprehension. Furthermore the presence of a simple speech was noticed in patients 3 and 6 . As regards AS dysmorphisms, only the wide 


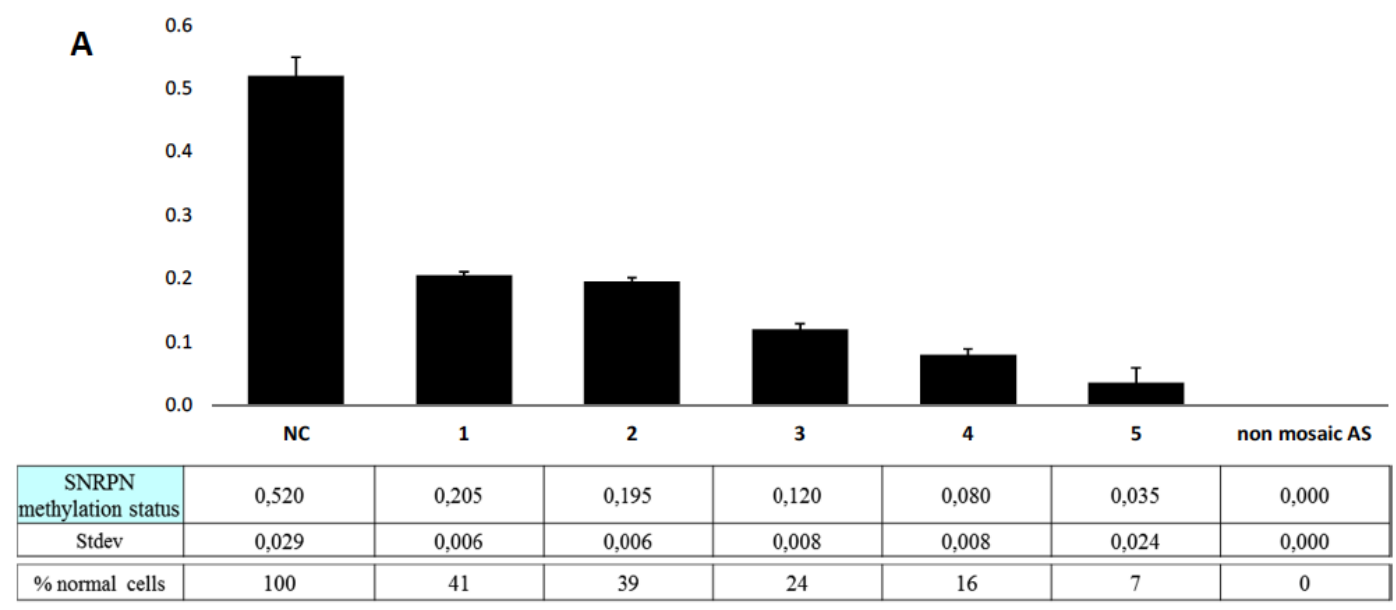

B

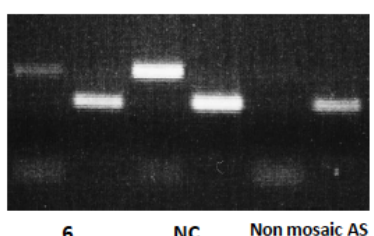

Figure 1: A) results of MS-MLPA test on cases 1 to 5 as compared to normal control (NC) and AS non mosaic epimutated cases. The methylation status of SNRPN locus with the standard deviation and the percentage of normal cells are indicated below, ordered from the higher to the lower methylation level; B) results of MS-PCR of case 6 , who could be investigated only by this method. A faint band for the maternal allele can be seen.
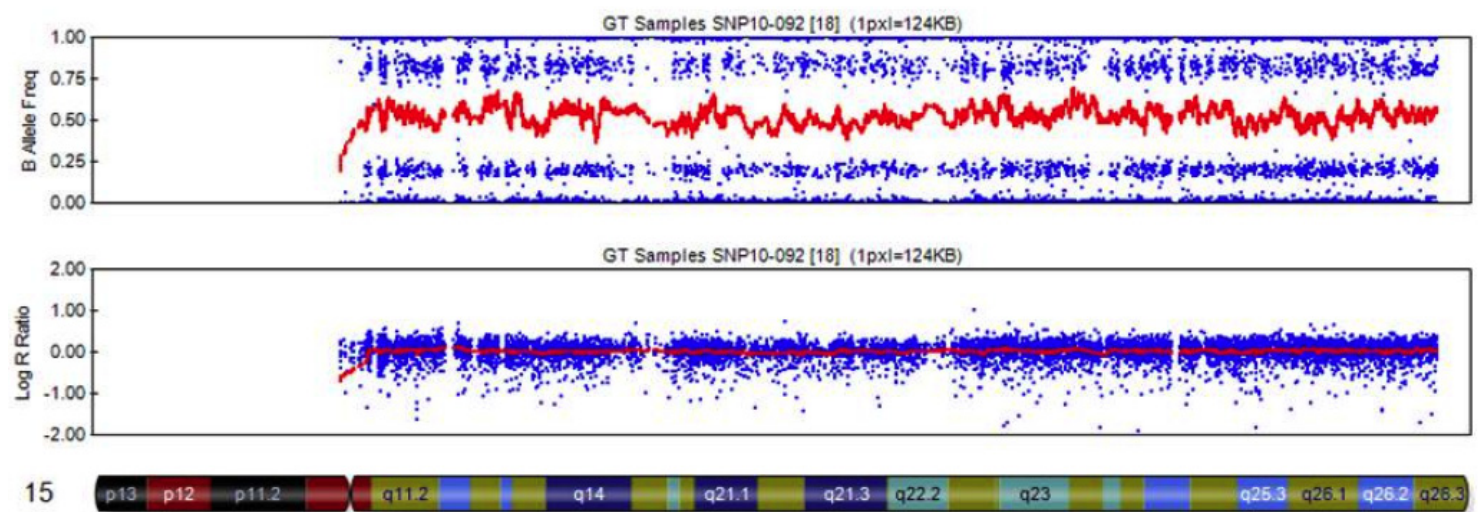

A

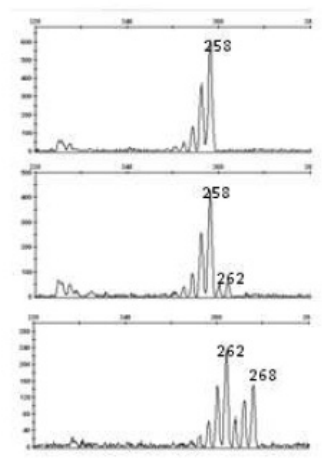

D $15 S 153$

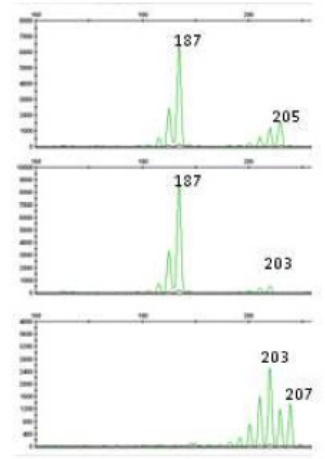

D 15 S 165

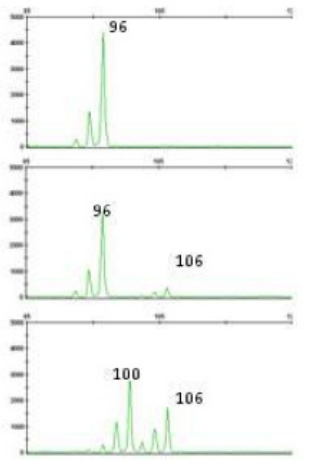

D 15 S1012

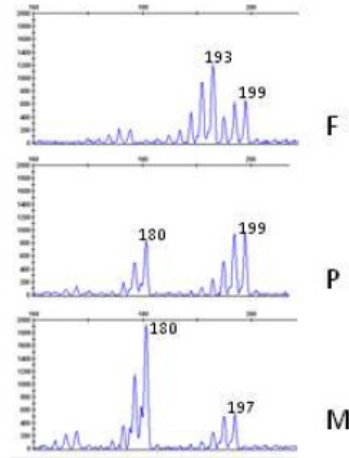

GABRB 3

Figure 2: Analysis of patUPD15 in case 2: A) Bead studio output shows the presence of mosaic Uniparental Disomy (UPD) for chromosome 15; B) the segregation analysis of microsatellites on chromosome 15 indicates the paternal origin of isodisomy and the presence of a limited percentage of maternal allele. 
Table 1: Clinical Features of the Mosaic ImpD and UPD Patients

\begin{tabular}{|c|c|c|c|c|c|c|c|c|}
\hline & MOSAIC PATIENTS & $1 \mathrm{ImpD}$ & 2 UPD & 3 ImpD & $4 \mathrm{ImpD}$ & $\begin{array}{c}5 \\
\operatorname{ImpD}\end{array}$ & $6 \mathrm{ImpD}$ & $\begin{array}{l}\text { non mosaic } \\
\text { UPD/ImpD[14] }\end{array}$ \\
\hline & Sex & M & M & $\mathrm{F}$ & $\mathrm{F}$ & M & $\mathrm{F}$ & $8 \mathrm{M} 5 \mathrm{~F}$ \\
\hline & Age at diagnosis & $4 y$ & $6 y$ & $6 y$ & $3 y 6 m$ & $6 y$ & $6 y$ & $0-60$ months \\
\hline & Age at last evaluation & $8 y$ & $6 y$ & $11 y$ & $8 y$ & $10 y$ & $20 y$ & $25-60$ months \\
\hline & Overweight & - & mild & mild & mild & - & - & + \\
\hline \multirow{5}{*}{$\begin{array}{l}\text { CONSISTENT } \\
\text { CRITERIA } \\
(100 \%)\end{array}$} & Intellectual Disability & $\begin{array}{c}\mathrm{IQ}=85 \\
\text { (Leiter-R, } \\
\text { 8y) IQ } \\
\text { Griffith } \\
61\end{array}$ & $\begin{array}{l}\text { IQ } \\
\text { Griffith } \\
68\end{array}$ & $\begin{array}{l}\text { IQT } \\
\text { Weschler } \\
<40\end{array}$ & mild & $\begin{array}{c}\text { no } \\
\text { scales }\end{array}$ & moderate & + \\
\hline & $\begin{array}{l}\text { Speech impairment: } \\
\text { minimal or no use of } \\
\text { words (onset of } \\
\text { speech) }\end{array}$ & $\begin{array}{l}4 \text { words } \\
\text { (first } \\
\text { words } \\
4 y \text { ) }\end{array}$ & + & $\begin{array}{l}\text { speech } \\
\text { delay, } \\
\text { simple } \\
\text { sentences }\end{array}$ & $\begin{array}{l}\text { ten words } \\
\text { (first words } \\
\text { at } 2 \mathrm{y} \text { ) }\end{array}$ & + & $\begin{array}{l}12 \text { words/ } \\
\text { some } \\
\text { complex } \\
\text { sentences }\end{array}$ & + \\
\hline & $\begin{array}{l}\text { Movement or balance } \\
\text { disorder (ataxia) }\end{array}$ & $\begin{array}{l}\text { very } \\
\text { mild }\end{array}$ & - & - & clumsiness & $\begin{array}{l}\text { very } \\
\text { mild } \\
\text { (on } \\
\text { tiptoes) }\end{array}$ & $\begin{array}{l}\text { on tiptoes } \\
\text { until } 3-4\end{array}$ & $8 / 11(73 \%)$ \\
\hline & Age of walking & $\begin{array}{c}14 \\
\text { months }\end{array}$ & $\begin{array}{c}10 \\
\text { months }\end{array}$ & 15 months & 18 months & $\mathrm{nr}$ & 14 months & $>2$ years \\
\hline & $\begin{array}{l}\text { AS behavior: frequent } \\
\text { laughter, hyperactivity }\end{array}$ & + & + & + & + & $\mathrm{nr}$ & + & + \\
\hline \multirow{3}{*}{$\begin{array}{l}\text { FREQUENT } \\
\qquad(>80 \%)\end{array}$} & Microcephaly & - & - & - & - & $\mathrm{nr}$ & + & $8 / 13(62 \%)$ \\
\hline & Abnormal EEG & + & + & + & + & + & + & + \\
\hline & Seizure and age of onset & +/3 year & - & - & - & - & +/7years & $6 / 13(46 \%)$ \\
\hline \multirow{17}{*}{$\begin{array}{l}\text { ASSOCIATED } \\
(20-80 \%)\end{array}$} & Flat occiput & - & - & - & + & - & - & $+/-$ \\
\hline & Occipital groove & - & - & - & - & - & - & $+/-$ \\
\hline & Protruding tongue & + & - & - & + & - & + & $+/-$ \\
\hline & Tongue thrusting & - & - & - & $+/-$ & - & - & $+/-$ \\
\hline & Prognatia & + & - & - & - & - & - & $3 / 13(23 \%)$ \\
\hline & $\begin{array}{l}\text { Wide mouth/wide spaced } \\
\text { teeth }\end{array}$ & $+/-$ & + & - & + & + & + & $9 / 13(69 \%)$ \\
\hline & Frequent drooling & + & - & - & + & - & - & $10 / 13(77 \%)$ \\
\hline & Excessive chewing & - & - & - & - & - & - & $11 / 13(85 \%)$ \\
\hline & Strabismus & + & - & $\begin{array}{l}\text { other eye } \\
\text { defects }\end{array}$ & - & - & - & $+/-$ \\
\hline & Hypopigmentation & - & - & - & - & - & + & $3 / 13(23 \%)$ \\
\hline & Hyperphagia & - & mild & mild & - & - & - & + \\
\hline & $\begin{array}{l}\text { Hyperactive lower limbs, } \\
\text { deep tendon reflexes }\end{array}$ & + & - & - & - & - & - & $\mathrm{nr}$ \\
\hline & Truncal/limbs hypotonia & $+/+$ & $\mathrm{nr}$ & $+/-$ & - & $\mathrm{nr}$ & + & $0 / 14$ \\
\hline & $\begin{array}{l}\text { Flexed arm position } \\
\text { during deambulation }\end{array}$ & - & - & - & - & - & - & $+/-$ \\
\hline & $\begin{array}{l}\text { Increased sensitivity to } \\
\text { pain }\end{array}$ & - & - & - & + & - & - & $\mathrm{nr}$ \\
\hline & Sleep disturbance & + & - & - & - & $\mathrm{nr}$ & - & $12 / 13(92 \%)$ \\
\hline & $\begin{array}{l}\text { Attraction/fascination } \\
\text { with water }\end{array}$ & + & - & - & - & $\mathrm{nr}$ & + & $8 / 13(62 \%)$ \\
\hline
\end{tabular}

nr: not reported. Clinical signs displayed by all cases are in bold. 


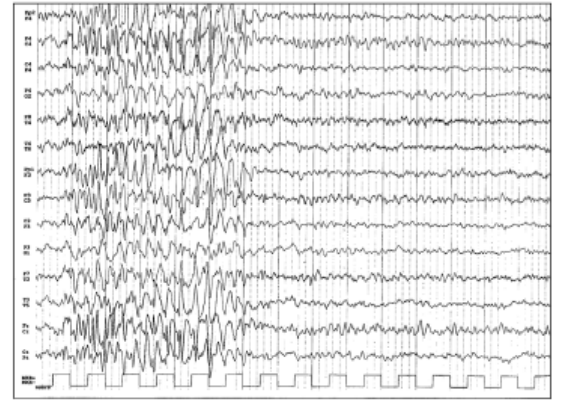

A

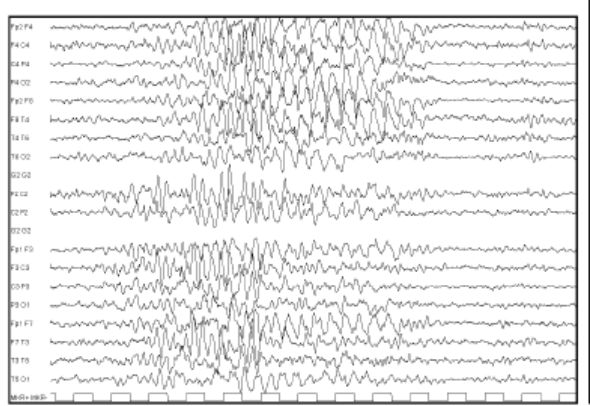

B

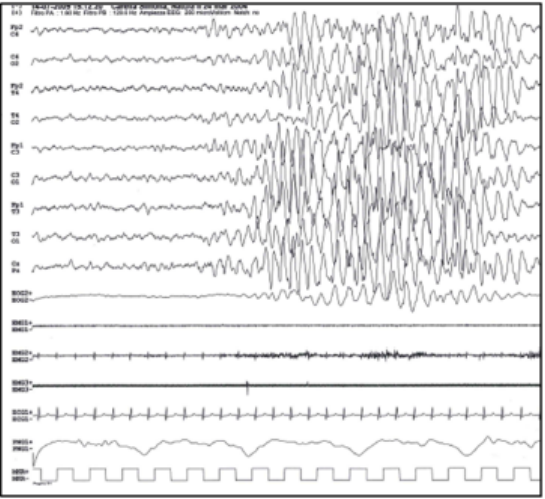

C

Figure 3: EEG of the cases 1(A), 2(B) and 3(C). A) Discharge of slow waves and spikes on fronto-temporal regions and vertex, B) discharges of high-amplitude slow waves on bilateral fronto-central and temporal regions with spike activity more evident on the right hemisphere, C) discharges of diffuse high-amplitude slow (theta) and sharp waves.

mouth appears as recurrent feature, being present in 5 out of 6 cases. On the other hand EEG shows the presence of generalized or anterior bilateral slow and spike waves of high amplitude in all patients, as shown for the representative cases in Figure 3 . The typical EEG, together with intellectual disability, speech impairment and characteristic AS hyperactive behavior represent the shared signs of the six patients (bolded in Table 1) all manifesting an atypical AS phenotype with a mild expression of the disease.

However, they appear quite different each other in their clinical features, as detailed by their clinical reports.

\section{Patient 1}

The child was born at term by a normal delivery $\left(41^{\circ}+4\right.$ weeks $)$ to non consanguineous parents. Birth weight was $3880 \mathrm{gr}$. Hypospadia was detected and corrected by surgery. Facial dysmorphisms are detailed in Table 1. At 8 months wall eye became evident. First milestones were correctly achieved with autonomous walking at 14 months. At 18 months he started to produce sounds, but he did not bubble and never learned to speak. At 30 months the child was referred for a neuropsychiatrist counselling because of speech delay. At the time of the first consultation he had a mild psychomotor delay (Griffiths' total score was 59 at 3 years) (Figure $\mathbf{5 A}$ ) and he used communication through gesture and pointing. The neurological examination revealed macrocrania $(>97 \%$ percentile), mild hypotonia, global motor impairment and mild dyspraxia, mild ataxia, discrete tremor of the fingers. He had an happy disposition, smiled frequently and had paroxysm of easily provoked laugher. He had sleep disturbance, attraction of water and a short attention span. EEG shows slow waves morphology discharge and spikes on frontal, temporal regions and vertex (Figure $3 \mathbf{A}$ ). Onset of focal polymorphic seizures occurred at 3 years. Therapy with Sodium valproate and Ethosuximide is maintained and he did not have seizures since 2010. He was submitted to Angelman methylation analysis about one year after seizure onset, when karyotype anomalies and fragile $X$ syndrome had been excluded.

Leiter-R battery reported a normal nonverbal IQ (87) at $4 y 9 \mathrm{~m}$ (visual functioning 87 , reasoning 96, communication 81 , forward memory 81 ). Parallely VABS was administered to the mother at $4 \mathrm{y} 9 \mathrm{~m}$ obtaining an equivalent age of 18 months for communication, 31 for daily living skills, 28 for social domains, 32 for motor skills. Verbal comprehension was assessed by TROG-2; he obtained low score $\left(<1^{\text {st }}\right.$ percentile), mainly because of short attention span. He has been treated for one month with methylphenidate without significant improvements, so the treatment was discontinued.

Actually the boy has finished the third grade; tremor of the hands impairs his fine motor skill, but he shows a high level of comprehension and willingness to learn. He can not speak, but he is able to read and write simple sentences. As shown in Figure $1 \mathrm{~A}$ he has $41 \%$ of normal cells in peripheral blood.

\section{Patient 2}

The male proband is the first child of a non consanguineous Italian mating. He was born at term by normal delivery and showed a birth weight of $3540 \mathrm{gr}$ (50th centile). He sat alone at 6 months and walked independently at 10 months. He is highly hyperactive 
and he has been treated for two years with methylphenidate. At age 6 the boy was referred for genetic counselling because of absence of speech. Audiometry assessment was normal. At clinical examination he showed short philtrum $(1 \mathrm{~cm}$; 3th centile) and hypertelorism (interpupillary distance is $6.63 \mathrm{~cm}$; >97th). The height $(125 \mathrm{~cm})$ was between 50 75th centile, head circumference $(52 \mathrm{~cm})$ was at the 50 th centile, instead the weight $(37 \mathrm{~kg})$ was above the 97th centile. No major dysmorphisms were evidenced, although a happy puppet face could have been suspected (Figure 4A). Karyotype analysis and SNP array were carried on without the clinical suspicious of Angelman syndrome. After molecular diagnosis was achieved, EEG showed discharge of slow waves and spikes on fronto-temporal regions and vertex (Figure 3B). Griffith scale at the age of $5 \mathrm{y} 4 \mathrm{~m}$ gave a total score of 68 , which corresponds to an age of 3 years and 1 month: partial scores are reported in Figure 5B. Verbal comprehension is low and when measured with the TROG test is below the first centile. He shows in blood $39 \%$ of normal cells.

\section{Patient 3}

Born to non consanguineous parents, the girl is the second child of three sibs with two healthy brothers. No significant family history was reported. The pregnancy was ordinary, birth weight was $3510 \mathrm{gr}$, length $52 \mathrm{~cm}$, head circumference $34,5 \mathrm{~cm}$. Motor skills were regularly achieved, while speech delay appeared evident at 4 years; at that age it was associated to a psychomotor development delay. The neurological examination revealed a mild hypotonia and global motor impairment, absence of ataxia, but orobuccal dyspraxia, motor and ideational dyspraxia and echopraxia were the dominant symptoms.
Serial neuropsychological assessments evidenced a moderate to severe degree of mental impairment. At 5 years the girl showed a mental deficit (total I.Q: 50 ) with a specific language impairment; at 7 years the IQ was stable, language was more compromised and difficulties of attention became evident.

WISC-IV test carried on at 9 years revealed a total $\mathrm{IQ}<40$ with scores for subtest corresponding to 50 for verbal comprehension, 45 for perceptual reasoning, 52 for working memory and 53 for processing speed: cognitive impairment ranged from moderate to severe with difficulties in both verbal and visual spatial tasks.

At the last follow-up (11 years) cognitive assessment attests a severe delay (Raven CPM: $+10 / 36$ correct answers- mental age $4 / 4 \mathrm{y} 6 \mathrm{~m}$ ). Visual perceptual and graphic tasks are also abnormal (VMI development test of visual motor integration -coding p.p. 1: p.s. 55 -mental age $4 \mathrm{y} 6 \mathrm{~m}-)$ and show severe learning disabilities (the patient is not able to read bisillabic words, but only to write her name and read alphabetic letters). Neuro-linguistic assessment shows severe deficits of expressive and receptive language characterized by phonological, lexical and morphosyntactic difficulties in both production and comprehension (receptive vocabulary WPPSI-III: mean for children $5 \mathrm{y} 2 \mathrm{~m}$ years old and picture naming WPPSI-III gave a mean score for children less than $2 \mathrm{y} 6 \mathrm{~m}$ old). In particular, the patient typically is able to produce sentences with two or three words that are used in functional way and for request; her receptive level is characterized by verbal comprehension of daily routine words and very short sentences (simple orders). Adaptive functioning is significantly delayed as she shows poor daily living skills (self-care activities, personal hygiene, dressing...). She has never had

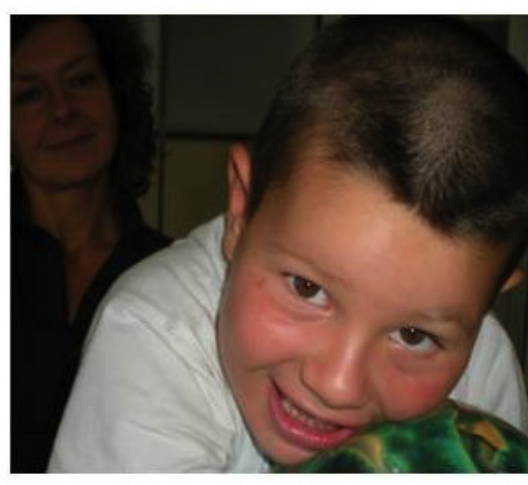

A

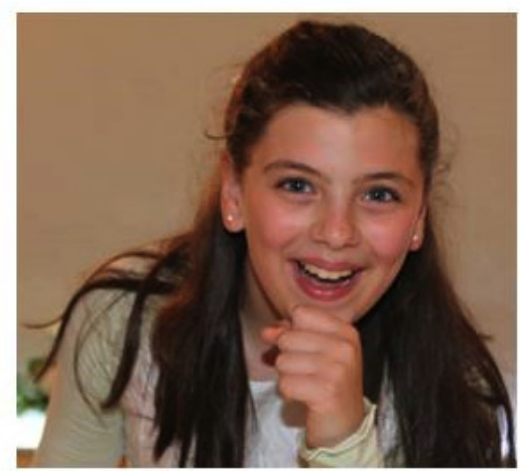

B

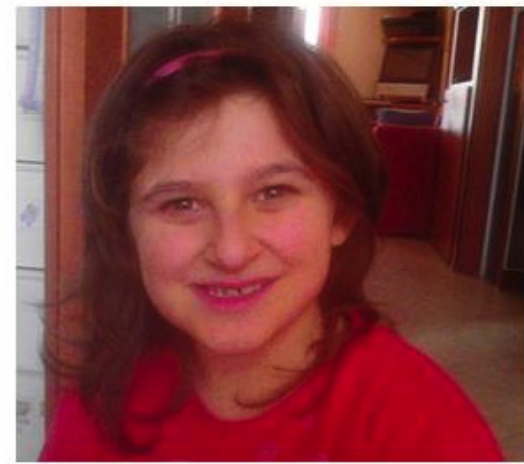

C

Figure 4: Faces of cases $\mathbf{2}(\mathbf{A}), \mathbf{3 ( B )}$ and $\mathbf{4}(\mathbf{C})$. The absence of peculiar dysmorphisms is evident in $\mathbf{A}$ and $\mathbf{B}$, while mild AS features are apparent in $\mathbf{C}$. 
A

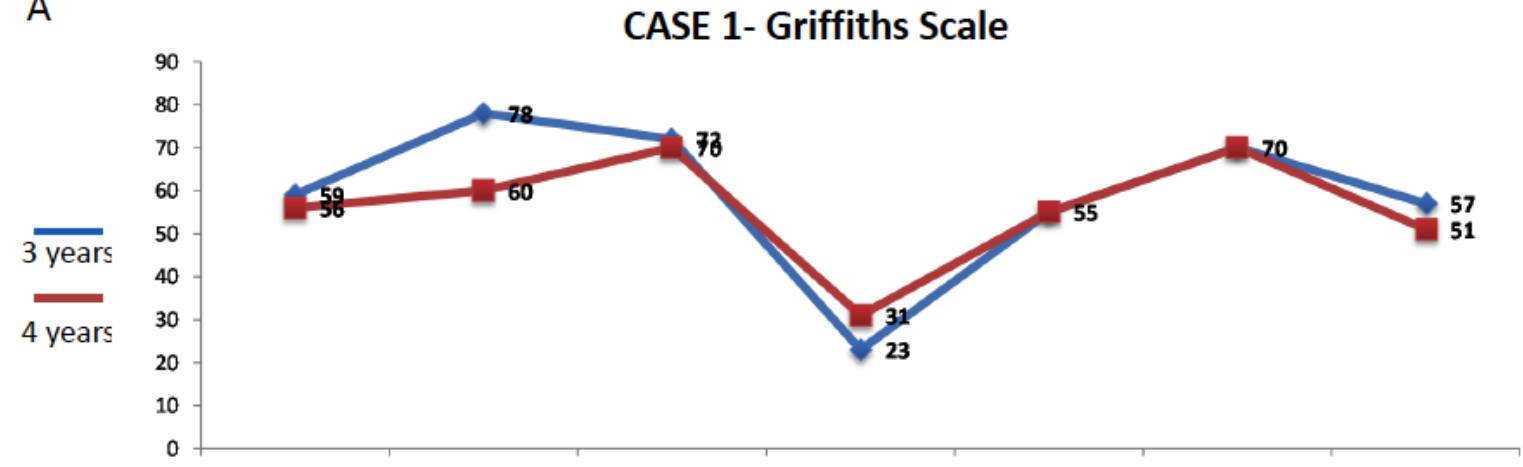

B
5 years
4 months

CASE 2- Griffiths Scale

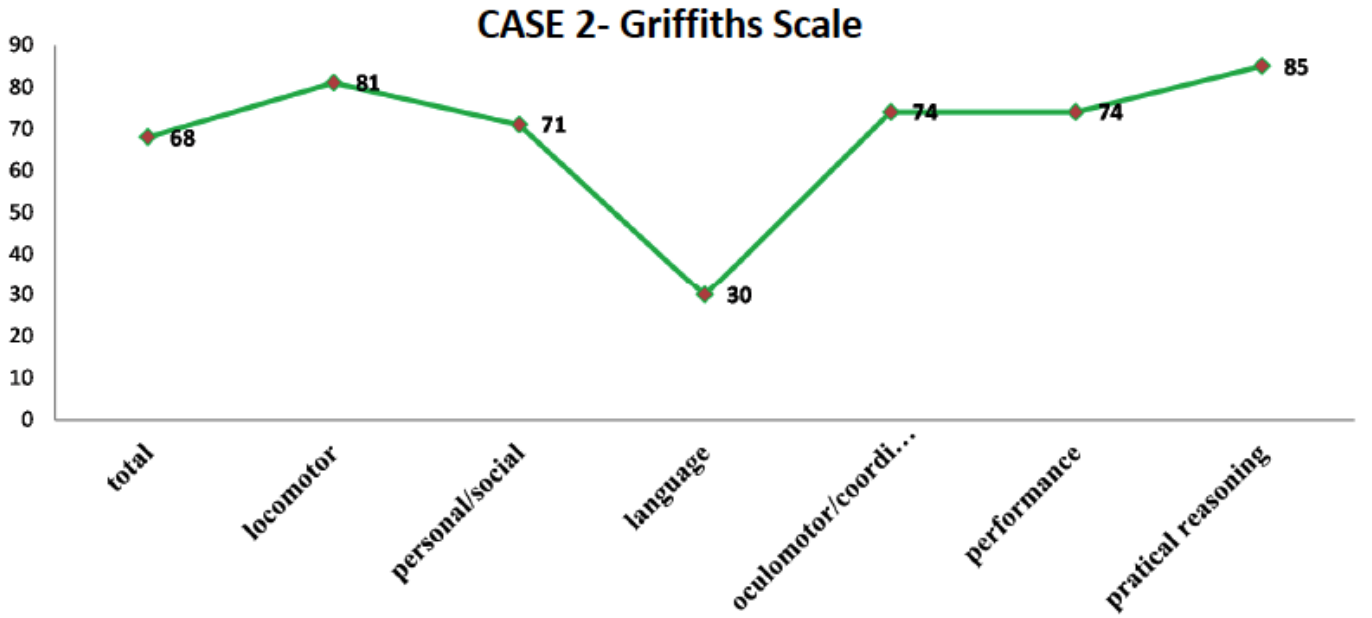

Figure 5: Partial scores testing different areas of the intellectual skills in cases $1(\mathbf{A})$ and $\mathbf{2 ( B )}$. The profile of the curve appears very similar.

seizures and never taken antiepileptic drugs, but her EEG recording showed discharges of diffuse highamplitude slow and sharp waves, still present at the last follow-up (Figure $\mathbf{3 C}$ ). No even mild facial dysmorphisms can be appreciated in the girl (Figure 4B).

The patient was addressed to molecular test only at 6 years, after chromosome abnormalities and fragile $X$ syndrome were excluded. Methylation analysis revealed a mosaic state with $24 \%$ of normal cells (Figure 1A)

\section{Patient 4}

The girl was submitted to molecular test for mild psychomotor delay, ataxia and speech delay. Facial traits are suggestive for Angelman syndrome, because of flat occiput, tongue trusting and wide mouth with spaced teeth (Figure 4C). She walked at 18 months and shows clumsiness. Her first words appeared at 2 years and consist of ten words. She never had seizures, but EEG showed the typical pattern of AS. She shows frequent laughter, easily excitable personality and hypermotoric behavior.

\section{Patient 5}

The boy was referred to our lab for absence of speech and mild psychomotor delay at age 6 . He was born to non consanguineous parents, family history was uneventful and delivery unremarkable. His facies did not resemble that of Angelman syndrome patients, apart from wide mouth and spaced teeth. EEG was typical. His mother did not remember exactly the age of walking, but at age of 10 years he was only mildly ataxic and walked on toes. Mosaicism was detected both by MS-MLPA and by MS-PCR. In this case only about $7 \%$ of cells were normal in blood.

\section{Patient 6}

The girl had an uneventful family history and a normal delivery at term. A severe gastroesophageal reflux during the first 2 months impaired breast feeding until 4 months. The girl achieved her developmental milestones quite regularly, walking at 14 months, but speech was almost absent. She has blond hair, bright skin and displays wide mouth as the only AS typical dysmorphism. Ataxia has not been reported, but she walked on toes. She was screened at 6 years by AS 
methylation test which showed a result of AS mosaic. At 7 years, she manifested the first seizures as generalized seizures and absences. EEG was typical of AS syndrome. Happy behavior was reported. At 20 years the girl speaks at least 12 words and makes efforts to mimick some complex sentences. Her learning disabilities are considered moderate by clinicians. In this patient mosaicism could be evaluated only by MS-PCR (Figure 1B), but her amount of maternal allele was similar to that of case 5 by MSPCR (data not shown) who has less than $10 \%$ of cells normal. Patients 4,5 and 6 were not assessed by intellectual disability scales.

\section{DISCUSSION}

It is assumed that genetic and environmental causes are the main determinants of ID, with genetic factors contributing to $25-50 \%$ of heritability $[1,2]$. However a large fraction, estimated up to $60 \%$, of syndromic and non syndromic ID patients remains without a known genetic cause. When the syndromic ID shows a defined specific presentation, clinical diagnosis is suspected and whenever the genetic mechanism is known, the patient easily achieves the diagnosis and the proper follow-up. Conversely, it may happen that only a few symptoms of a specific syndrome are recognizable or that they are only mildly expressed, hampering the clinical recognition and diagnosis.

Our study exemplifies the occurrence of cellular mosaicism as a relevant factor able to contribute to the phenotypic heterogeneity and to modulate the clinical presentation of the well known Angelman imprinting disorder [26]. According to our data more than 1 every four cases of AS cases with an ImpD defect carries the epimutation in a mosaic condition, accounting for the usually delayed clinical recognition of the syndromic phenotype in these cases. When manifested by its classic phenotype, AS is characterized by distinctive features, which constitute the major consensus criteria $[4,5]$ and define traits that should be present in all the patients, as the typical facies, the absence or almost of speech, a peculiar EEG and severe to moderate ID. It is known for AS that the different underlying molecular mechanisms correlate with the clinical phenotype $[13,14]$ and that patients carrying patUPD 15 and ImpD defects have a relatively milder clinical presentation as compared to deletion and UBE3A mutated carriers. Studies addressing genotype-phenotype correlations usually keep together the UPD and ImpD patient cohorts, as they appear quite homogeneous in the phenotypic expression. As expected, the main AS consensus criteria are manifested in these two patient subsets, but their degree of expression is less pronounced, while minor criteria are observed in a variable percentage of patients. As a result of mosaicism the mild clinical phenotype might be even milder or may feature as atypical, hampering the recognition of the syndrome. Our small cohort confirms a rate of mosaicism in the ImpD group of $28 \%$, close to that $(31 \%)$ found by Nazlican [16]. Interestingly it also discloses the occurrence of mosaic patUPD15, which has never been reported for Angelman syndrome. Summing up our cases to those with a similar epigenotype previously reported [15-19] a total of about 35 mosaic epimutated cases have been clinically described. In our study the fraction in blood of normal cells ranges from 7 to $41 \%$. We argue that a major proportion of correctly methylated cells might be present in patients with a borderline phenotype, who are not submitted to AS methylation test and remain without a molecular diagnosis. Till MS-PCR and Southern Blot were the routine techniques to screen 15q11-q13 methylation defects, mosaic epimutations could be detected although without the quantitative assessment provided by the subsequent widespread application of MS-MLPA, which allowed us to identify a SNRPN methylation status of 0.2 , approximately corresponding to $40 \%$ of normal cells. Even if most of AS patients show a SNRPN methylation status between 0 and 0.1 we should keep into consideration the possibility that a progressively decreased level of epimutated cells may account for a spectrum of clinical signs displayed by patients with intellectual disability and a few subtle Angelman traits.

All cases herein presented merge with those in the literature in demonstrating that when the imprinting defect affects a limited proportion of cells, the expression of the symptoms is lessened. Consistent with other studies [15-19] we observed a better performance in motor coordination, being ataxia mildly present in two out of our six 6 cases and onset of walking recorded at the normal age for all our six children. Only two children have seizures and case 1 is still under treatment. Many speak only some words or simple sentences. The typical facies is not observed in our children, with the exception of wide mouth and spaced teeth only absent in case 3. Differently from Nazlican's patients, none of ours shows a Prader Willi phenotype and overweight was reported in cases 2, 3 and 4. Despite all mosaic patients display a phenotype milder than that of non mosaic ones, they are not 
clinically homogenous, as each has a few, not necessarily shared, mild signs of Angelman, thus making hard the diagnosis. This variability can be imputed to the variable level of mosaicism in different tissues, some of which, like the brain, are most sensitive to the defect, although not accessible.

Key features to be kept into account when the association of ID with speech delay raises the suspicion of AS and addresses the patient to methylation test of 15q11-13 region, are the EEG, which often appears characteristic for the syndrome, the happy behavior and the presence of a receptive language much more developed than the expressive one (see in Table 1 clinical signs displayed by all patients). Cognitive scales were administered (with different tools, because the patients were diagnosed and have their follow up in different hospitals) to cases $1,2,3$ who have the major proportion of normal cells. Almost all the scales attested a mild mental retardation, with significant difficulties in language expression, but only mildly impaired performance skills. Case 1 obtained a normal score at Leiter- $R$, that measures non verbal intelligence regardless of motor and expressive or receptive language level. However at Griffith scale, characterized by items that require linguistic and motor skills and investigating domains which affect personal and social autonomies development, case 1, despite his normal non verbal intelligence, has an adaptive functioning delay (Figure 5A). Interestingly a similar Griffith profile is shared by case 2 who has a comparable amount of normal cells (Figure 5B).

Last we report for the first time on a case of mosaic uniparental disomy of paternal chromosome 15. Usually paternal UPDs are commonly originated by monosomy rescue $[27,28]$. The occurrence of mosaicism with correct biparental genotype in some cells and paternal isodisomy in the remaining cells (see Figure 2B) indicates a post-fertilization mechanism implying an event of mitotic replication error, leading to the loss of maternal chromosome 15 and duplication of paternal homolog. SNP array is confirmed to be a very sensitive tool in detecting low level mosaicism.

To conclude, we can speculate that a major proportion of correctly methylated cells, $>20-40 \%$ according to this study, is present in patients with an unexpected phenotype, not submitted to AS methylation test. We believe that one should keep into consideration the possibility that lower mosaicism rates may account for a spectrum of clinical signs displayed by patients with intellectual disability and a few Angelman traits.

\section{ACKNOWLEDGEMENT}

We thank the Italian parents' association OrSA for their availability, cooperation and support for publication. This work was supported by Istituto Auxologico Italiano, research 08C002_2010.

\section{REFERENCES}

[1] Karam SM, Riegel M, Segal SL, et al. Genetic causes of intellectual disability in a birth cohort: A population-based study. Am J Med Genet A 2015; 167(6): 1204-1214. http://dx.doi.org/10.1002/ajmg.a.37011

[2] Kaufman L, Ayub M, Vincent JB. The genetic basis of nonsyndromic intellectual disability: a review. J Neurodevelop Disord 2010; 2: 182-209. http://dx.doi.org/10.1007/s11689-010-9055-2

[3] Ropers $\mathrm{HH}$. X-linked mental retardation: many genes for a complex disorder. Curr Opin Genet Dev 2006; 16(3): 260269.

http://dx.doi.org/10.1016/j.gde.2006.04.017

[4] Williams CA, Angelman H, Clayton-Smith J, et al. Angelman syndrome: consensus for diagnostic criteria. Angelman Syndrome Foundation. Am J Med Genet 1995; 56(2): 237238.

http://dx.doi.org/10.1002/ajmg.1320560224

[5] Williams CA, Beaudet al, Clayton-Smith J, et al. Angelman Syndrome 2005: updated consensus for diagnostic criteria. Am J Med Genet A 2006; 140(5): 413-418. http://dx.doi.org/10.1002/ajmg.a.31074

[6] Kishino T, Lalande M, Wagstaff J. UBE3A/E6-AP mutations cause Angelman syndrome. Nat Genet 1997; 15(1): 70-73. http://dx.doi.org/10.1038/ng0197-70

[7] Dagli A, Buiting K, Williams CA. Molecular and clinical aspects of Angelman syndrome. Mol Syndromol 2012; 2(35): $100-112$

[8] Buiting K, Clayton-Smith J, Driscoll DJ, et al. Clinical utility gene card for: Angelman Syndrome. Eur J Hum Genet 2014; 23(2).

http://dx.doi.org/10.1038/ejhg.2014.93

[9] Dindot SV, Antalffy BA, Bhattacharjee MB, Beaudet al. The Angelman syndrome ubiquitin ligase localizes to the synapse and nucleus, and maternal deficiency results in abnormal dendritic spine morphology. Hum Mol Genet 2008; 17(1): 111-118.

http://dx.doi.org/10.1093/hmg/ddm288

[10] Yashiro K, Riday TT, Condon $\mathrm{KH}$, et al. Ube3a is required for experience-dependent maturation of the neocortex. Nat Neurosci 2009; 12(6): 777-783. http://dx.doi.org/10.1038/nn.2327

[11] Mabb AM, Judson MC, Zylka MJ, Philpot BD. Angelman syndrome: insights into genomic imprinting and neurodevelopmental phenotypes. Trends Neurosci 2011; 34(6): 293-303

http://dx.doi.org/10.1016/j.tins.2011.04.001

[12] Lossie AC, Whitney MM, Amidon D, et al. Phenotypes distinguish the molecular classes of Angelman syndrome. J Med Genet 2001; 38(12): 834-845.

http://dx.doi.org/10.1136/jmg.38.12.834

[13] Gentile JK, Tan WH, Horowitz LT, et al. A Neurodevelopmental survey of Angelman syndrome with genotype-phenotype correlations. J Dev Behav Pediatr 2010; 31(7): 592-601. http://dx.doi.org/10.1097/dbp.0b013e3181ee408e

[14] Tan WH, Bacino CA, Skinner SA, et al. Angelman syndrome: Mutations influence features in early childhood. Am J Med Genet A 2010; 155A(1): 81-90. 
[15] Gillessen-Kaesbach G, Demuth S, Thiele $\mathrm{H}$, et al. A previously unrecognised phenotype characterised by obesity, muscular hypotonia, and ability to speak in patients with Angelman syndrome caused by an imprinting defect. Eur $\mathrm{J}$ Hum Genet 1999; 7(6): 638-644. http://dx.doi.org/10.1038/sj.ejhg.5200362

[16] Nazlican H, Zeschnigk $M$, Claussen $U$, et al. Somatic mosaicism in patients with Angelman syndrome and an imprinting defect. Hum Mol Genet 2004; 13(21): 2547-2555. http://dx.doi.org/10.1093/hmg/ddh296

[17] Camprubí C, Coll MD, Villatoro S, et al. Imprinting center analysis in Prader-Willi and Angelman syndrome patients with typical and atypical phenotypes. Eur J Med Genet 2007; 50(1): 11-20.

http://dx.doi.org/10.1016/j.ejmg.2006.10.001

[18] Lawson-Yuen A, Wu BL, Lip V, Sahoo T, Kimonis V. Atypical cases of Angelman syndrome. Am J Med Genet A 2006; 140(21): 2361-2364. http://dx.doi.org/10.1002/ajmg.a.31481

[19] Fairbrother LC, Cytrynbaum C, Boutis P, Buiting K, Weksberg R, Williams C. Mild Angelman syndrome phenotype due to a mosaic methylation imprinting defect. Am J Med Genet A 2015; 167(7): 1565-1569. http://dx.doi.org/10.1002/ajmg.a.37058

[20] Zilina O, Kahre T, Talvik I, Oiglane-Shlik E, Tillmann V, Ounap K. Mosaicism for maternal uniparental disomy 15 in a boy with some clinical features of Prader-Willi syndrome. Eur J Med Genet 2014; 57(6): 279-283.

http://dx.doi.org/10.1016/i.ejmg.2014.03.007
[21] Wechsler D. Wechsler Adult Intelligence Scale - 3rd ed. San Antonio: The Psychological Corporation; 1997.

[22] Roid GH, Miller LJ. Leiter-R: Leiter International Performance Scale - Revised. Firenze: Giunti OS Edizioni Speciali; 1997.

[23] Griffiths R. The Abilities of Young Children. ARICD: Amersham; 1984.

[24] Sparrow SS, Cicchetti DV, Balla DA. Vineland Adaptive Behavior Scales: 2nd Edition. Livonia: Pearson Assessments; 2005.

[25] Kubota T, Das S, Christian SL, Baylin SB, Herman JG Ledbetter DH. Methylation-Specific PCR Simplifies Imprinting Analysis. Nat Genet 1997; 16(1): 16-17.

[26] Saitoh S, Buiting K, Rogan PK, et al. Minimal definition of the imprinting center and fixation of a chromosome 15q11-q13 epigenotype by imprinting mutations. Proc Natl Acad Sci USA 1996; 93(15): 7811-7815. http://dx.doi.org/10.1073/pnas.93.15.7811

[27] Liehr T. Cytogenetic contribution to uniparental disomy (UPD). Mol Cytogenet 2010; 3: 8 . http://dx.doi.org/10.1186/1755-8166-3-8

[28] Robinson WP. Mechanisms leading to uniparental disomy and their clinical consequences. BioEssays 2000; 22(5): 452459.

http://dx.doi.org/10.1002/(SICl)15211878(200005)22:5<452::AID-BIES7>3.0.CO; $2-\mathrm{K}$

\section{DOI: http://dx.doi.org/10.6000/2292-2598.2015.03.03.2}

(C) 2015 Russo et al.; Licensee Lifescience Global.

This is an open access article licensed under the terms of the Creative Commons Attribution Non-Commercial License (http://creativecommons.org/licenses/by-nc/3.0/) which permits unrestricted, non-commercial use, distribution and reproduction in any medium, provided the work is properly cited. 